\title{
Personality Prediction Using THROUGH CV ANALYSIS
}

\author{
MuthuselviM, Angeline Ranjitha Mani and AbinayaV \\ Department of Computer Applications, Francis Xavier Engineering College, \\ Tirunelveli
}

\begin{abstract}
This Human Resource Management is obviously bolstered by and gave more open doors by the improvement of Job Characteristics Model (JCM) which thusly depends on the idea of present day occupation plan. Luckily, the advancement in present day data framework, computerized innovations, the general access of electronic innovation and web prompted the tendency of the worldwide Human Resource. The board improvement and make the framework more pertinent. Following the pattern, the proposed framework attempts to structure an arrangement to coordinate Job Characteristics Model into E-HR framework to scan for another model of proficient activity on Human Resource Management in the Internet Age. In this venture, we present a lot of strategies that makes the entirety enlistment process increasingly viable and productive. We have executed a framework that positions the competitors dependent on weight-age arrangement just as a bent test. Today there is a developing enthusiasm for the character attributes of an up-and-comer by the association to more readily look at and comprehend the competitor's reaction to comparable conditions. Along these lines, the framework directs a character expectation test to decide the character attributes of the applicant. At long last, it shows the consequences of the contender to the selection representative who assesses the top competitors and waitlists the applicant. This system can used in many business sectors that may require expert candidate. This system will reduce workload of the human resources. This system will help the human resource to select right candidate for desired job profile, which in turn provide expert workforce for the organization. Admin can easily shortlist a candidate based on their online test marks and select the appropriate candidate for particular job profile. This will enable a more effective way to short list submitted candidate CVs from a large number of applicants providing aconsistent and fair CV ranking policy, which can be legally justified
\end{abstract}

\section{KEYWORDS}

Personality, Service oriented architecture, CV ranking, Aptitude, Questions, Human Resource Management

\section{INTRODUCTION}

Job Characteristics Model (JCM) which thusly depends on the idea of present day occupation plan. Luckily, the advancement in present day data framework, computerized innovations, the general access of electronic innovation and web prompted the tendency of the worldwide Human Resource. The board improvement and make the framework more pertinent. Following the pattern, the proposed framework attempts to structure an arrangement to coordinate Job Characteristics Model into E-HR framework to scan for another model of proficient activity on Human Resource Management in the Internet Age.the association to more readily look at and comprehend the competitor's reaction to comparable conditions. Along these lines, the framework directs a character expectation test to decide the character attributes of the applicant. At long last, it shows the consequences of the contender to the selection representative who 
assesses the top competitors and waitlists the applicant. This system can used in many business sectors that may require expert candidate. This system will reduce workload of the human resources. This system will help the human resource to select right candidate for desired job profile, which in turn provide expert workforce for the organization. Admin can easily shortlist a candidate based on their online test marks and select the appropriate candidate for particular job profile. This will enable a more effective way to short list submitted candidate CV s from a large number of applicants providing aconsistent and fair CV ranking policy, which can be legally justified.

\section{LITERATURE SURVEY}

Afify (2011)Is highly related to the research topic as it presents a new model of job web service based on Service-Oriented Architecture (SOA). In this model, the researchers have tried to regain the connection between user and Social Solidarity. The proposed model aims to create a Webbased job and resume related to the subsidiary lifecycle. The cycle begins with the distribution of various services supplied through the subsidiary. Those services were delivered to various people according to their requirements. Due to different hindrances, those services may not be efficiently applied. Because of that, there has been a must for a system that tracks down and discovers user problems and provides them with suitable feedback. This system can manage complaints through recording them and giving feedback for each raised complaint. The study outcome has been a helpful reference to determine users' requirements from the job and the managing process of this complaint in the core of any firm.

Trappey (2010)Have analyzed the configuration of the personality handling system for a restaurant chain in Japan. The complaint handling process gets over the defective approach of prior complaint handling through modifying the operations. So, this was beneficial to the operations taking place between the chain main administrative center and the other branches. To describe the personality handling model and its process, the formal integrated process modelling approach has been utilized. The new configuration contained complaint reporting, compensation diagnosis as well as complaint analysis. Moreover, the model, through the system, can spontaneously give the decision support on complaint resolution.

TruptiBomble, RitikaRaut and RuchiKanekar (2015)Presented a paper on android based personality prediction from cv. This system proposes on developing an android application which will help the common people under the same jurisdiction of a municipal corporation to register their grievances about day to day problems in their locality through a mobile application, but the problem is that fake complaints can be entered and there is no possibility to verify before resolving it. In addition to this there is no guarantee regarding progress of every jobs. Our system overcomes these problems by, priority based complaint resolving method and every registered complaint will get an acknowledgement on the complaint with the due date of completion.

Ali Mohammad Ahmadvand and ElhamAkhondzadeh (2010)Presented a paper on effective user relationship management. In this they proposed a system on data mining the complaints made by the citizens to manage their requirements and a process which collects, analyzes, and evaluate their needs. The problem in this system is, that the system can't solve the day to day grievance of the citizens. But in our proposed system, the day to day grievance of citizen will be registered by the citizen and will be resolved in due time.

Pyon (2011)Have thought that personality by call centers are enough to support the analysis of service promotion in the monetary service industry. Thus, they have proposed a web-based decision support system for running the business operation exploiting customer complaints; Voice of the Customer (VOC). The system has been tackling information for service promotion 
and involving VOC conversion for information upgrading together with including analysis of outlining, exception, and comparisons.

\section{Problem Statements}

The existing e-recruitment system simply scans the submitted CVs and shortlist the candidates wherein the proposed system conducts an online aptitude test and personality test thereby predicting the personality of the candidate as well as short-listing the candidate based on his skills and decision-making ability.The impact of manual interviews and the resumes over HR has kept on increasing in recent years. It is very important to come up with a solution that can shorten or fasten the HR department work. Therefore a system has been implemented that recommends the candidates CV. Traditional forms of recruitment typically involve job seekers filling out physical resumes and giving interviews with the surge in applicants lately, the number of candidates tends to over whelm the employers. The proposed automated candidate grading system utilizes machine learning algorithms to build the models which test them.

Candidate can also give an online test, which will be conducted on personality questions as well as aptitude questions. After completing the online test, candidate can view their own test results in graphical representation with marks. The system would then derive and rank the candidates who were eligible for the job. The rank of each candidate acts as a score of how well the candidate's profile meets the specifications of the recruiters as well as cumulative score of the aptitude test. The recruiter could also analyze the personality of the candidate based on the result of the personality test. So, based on CV, aptitude test and the personality test the candidate would be shortlisted.

\section{Architecture Diagram}

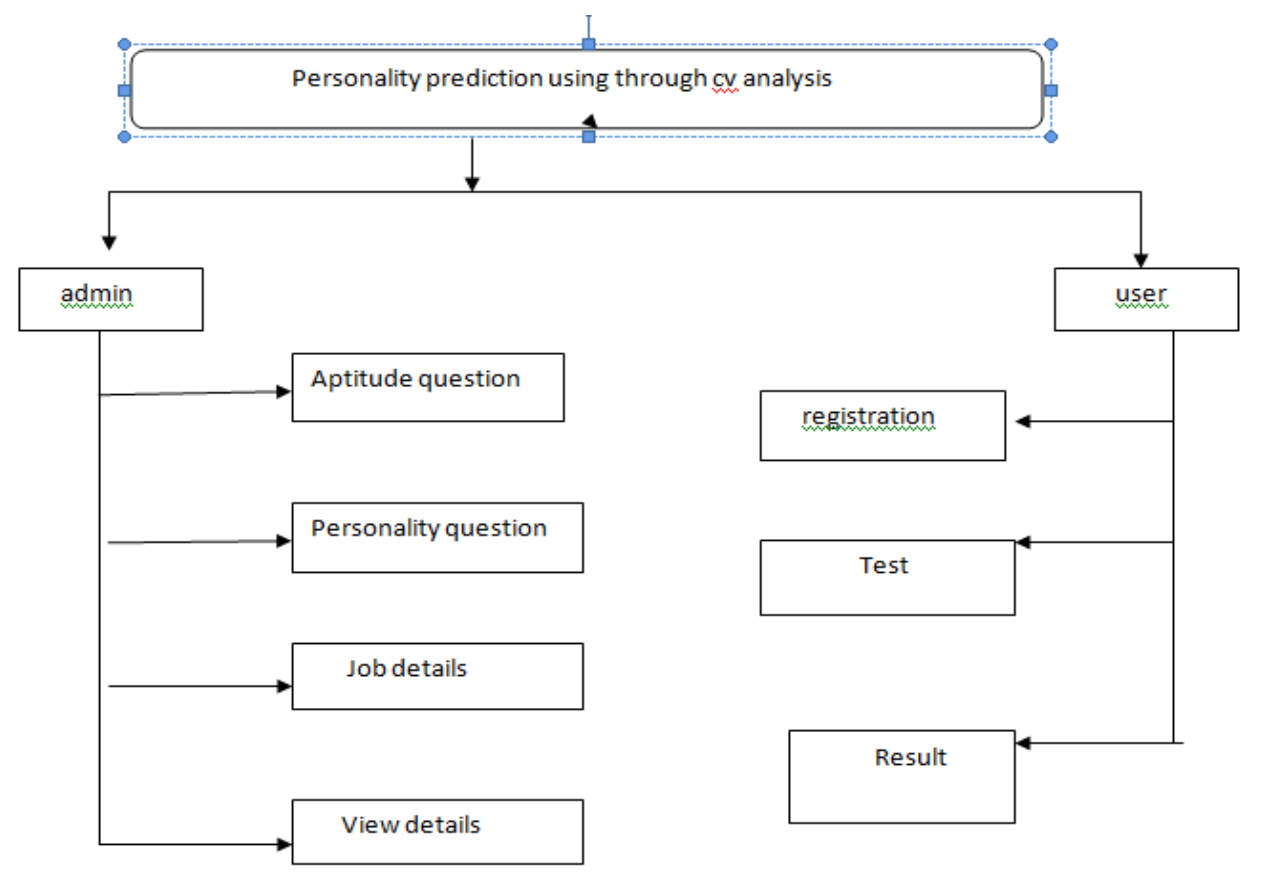




\section{FeASIBILity}

Preliminary investigation examine project feasibility, the likelihood the system will be useful to the organization. The main objective of the feasibility study is to test the Technical, Operational and Economical feasibility for adding new modules and debugging old running system. All system is feasible if they are unlimited resources and infinite time. There are aspects in the feasibility study portion of the preliminary investigation:

- Technical Feasibility

- Operational Feasibility

- Economical Feasibilty

\section{Technical Feasibility}

Earlier no system existed to cater to the needs of 'Secure Infrastructure Implementation System'. The current system developed is technically feasible. It is a web based user interface for audit workflow at NIC-CSD. Thus it provides an easy access to the users. The database's purpose is to create, establish and maintain a workflow among various entities in order to facilitate all concerned users in their various capacities or roles. Permission to the users would be granted based on the roles specified. Therefore, it provides the technical guarantee of accuracy, reliability and security. The software and hard requirements for the development of this project are not many and are already available in-house at NIC or are available as free as open source. The work for the project is done with the current equipment and existing software technology. Necessary bandwidth exists for providing a fast feedback to the users irrespective of the number of users using the system.

\section{* Operational Feasibility}

Proposed projects are beneficial only if they can be turned out into information system. That will meet the organization's operating requirements. Operational feasibility aspects of the project are to be taken as an important part of the project implementation. Some of the important issues raised are to test the operational feasibility.

\section{*conomic Feasibility}

A system can be developed technically and that will be used if installed must still be a good investment for the organization. In the economical feasibility, the development cost in creating the system is evaluated against the ultimate benefit derived from the new systems. Financial benefits must equal or exceed the costs. The system is economically feasible. It does not require any addition hardware or software.

\section{EXPERIMENTS AND RESULTS}

\section{- $\quad$ Aptitude Question}

This module was created for the added the aptitude question. The question can be a added only admin. The admin, who will be a question, will upload the questions into the database, which can be accessed by the authenticated users. The aptitude questionallowsthestudenttolearntheoutlineofthetopic, whichistobetaughtonthefore- coming class. 


\section{- Personality Question}

This module was created for the added the aptitude question. The question can be a added only admin. The admin, who will be a question, will upload the questions into the database, which can be accessed by the authenticated users. The aptitude questionallowsthestudenttolearntheoutlineofthetopic, whichistobetaughtonthefore- coming class. Give Test these types

The system interface is going to show the questions of different . The mainly going to

$>$ Maths

$>$ English

$>$ Programming

\section{- Take Test}

By using the user id and password user can log into the system and collect the necessary information from the home page.

$>$ Mathematical Question

$>$ English Question

$>$ Programming Question

$>$ Personality Question

$>$ Aptitude Question

\section{- Result For Chart}

The User can be result in the pages. For Example,

$>$ Chart way of English question

$>$ Chart way of Maths Question

$>$ Chart way of Programming Question

$>$ Chart way of Personality question

$>$ Chart way of Aptitude question

\section{- Database Collections}

The administrative user interface concentrates on the consistent information that is practically, part of the organizational activities and which needs proper authentication for the data collection. The interfaces help the administrations with all the transactional states like Data insertion, Data deletion and Data updating along with the extensive data search capabilities.The operational or generic user interface helps the users upon the system in transactions through the existing data and required services. The operational user interface also helps the ordinary users in managing their own information helps the ordinary users in managing their own information in a customized manner as per the assisted flexibilities

\section{About Project}

The major inputs and outputs and major functions of the system are follows:

Admin enter his user id and password for login. User enters his user id and password for login. Admin enter user id or date for track the user login information New users give his completed 
personnel, address and phone details for registration. Admin gives different kind of user information for search the user data. User gives his user id, hint question, answer for getting the forgotten password. User request for Municipal Bill. Admin can have his own home page.

Users enter their own home page. The user defined data can store in the centralized database. Admin will get the login information of a particular user. The new user's data will be stored in the centralized database. Admin get the search details of different criteria. User can get his forgot password. User can get the Bill Details.

\section{OUTPUT SCREEN}

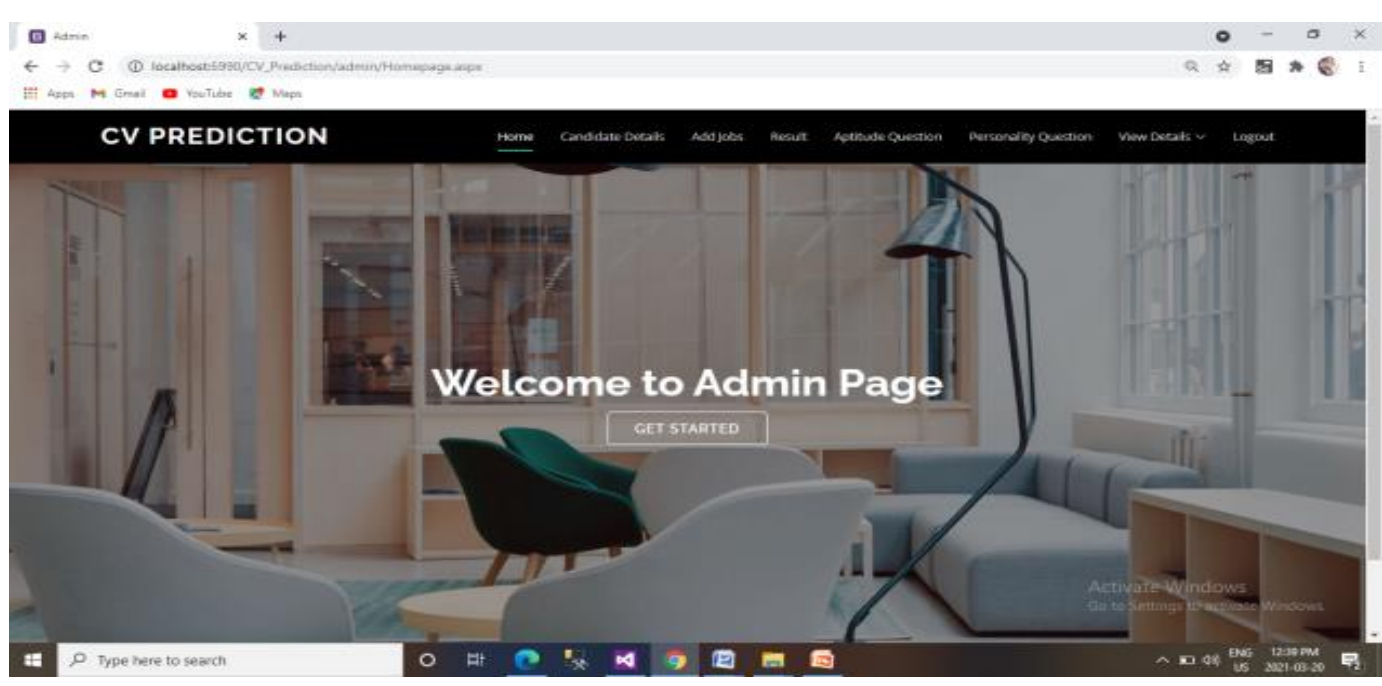

Figure: 1.1 - Home Page



User Login
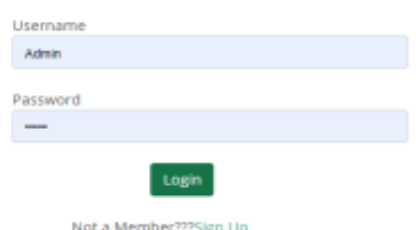

Figure: 1.2 - Login Page 

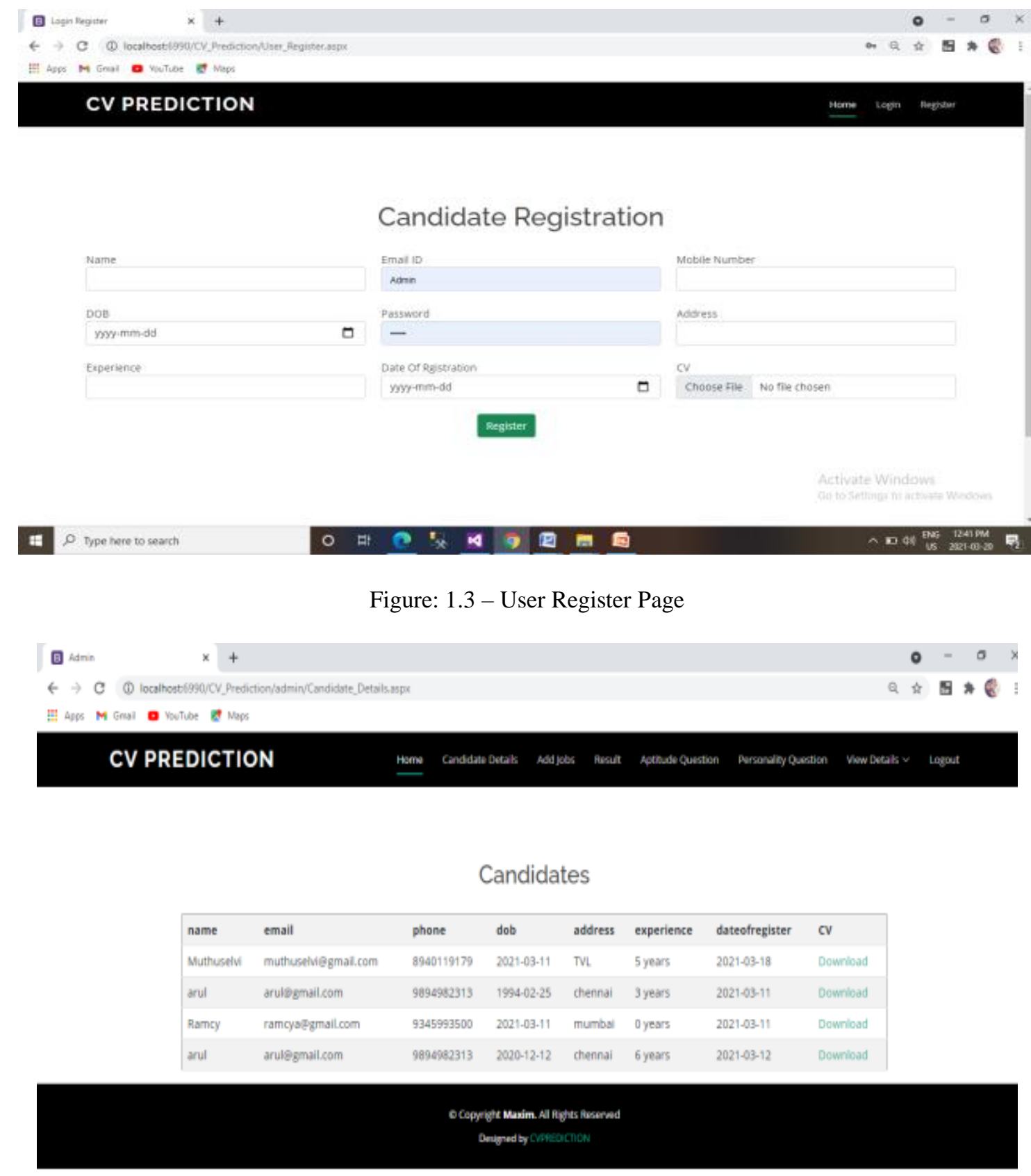

Figure: 1.4 - Candidate Details 


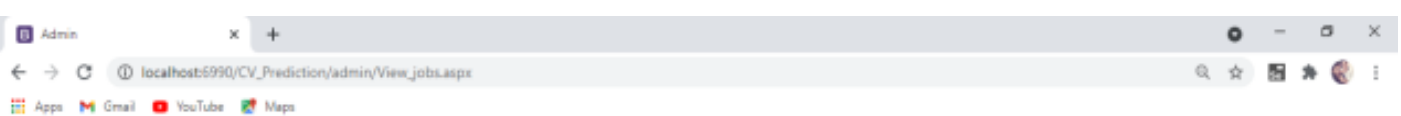

\begin{tabular}{|c|c|c|c|c|c|c|}
\hline \multicolumn{7}{|c|}{ Job Details } \\
\hline & id & technology & position & solery & experience & key_skills \\
\hline Delete & 1 & Dotnet & Developer & 500000 & 5 years & $C=$ Angular. \\
\hline Delete & 2 & python & developer & 30500 & 1 & Python, \\
\hline Delete & 3 & jwa & Developer & 40000 & 1 & Jaws, Python, \\
\hline
\end{tabular}

Figure: 1.5 - Job Details



Figure: 1.6 - Apptitude Question



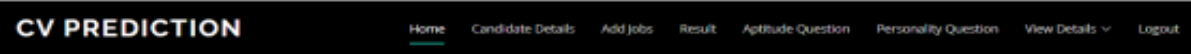

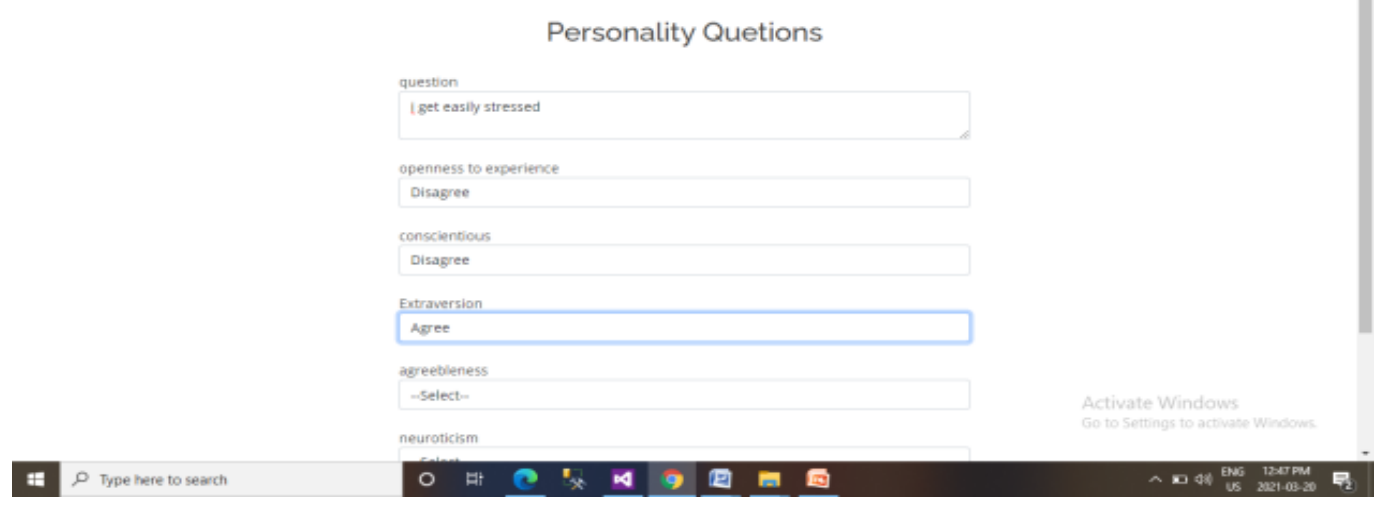

Figure: 1.7 - Add A Personality Question 


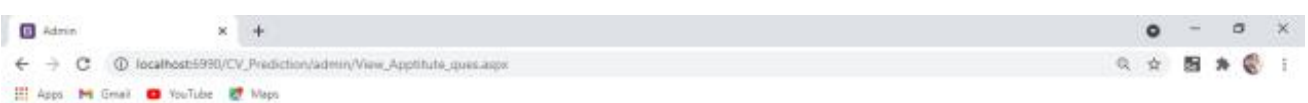

CV PREDICTION



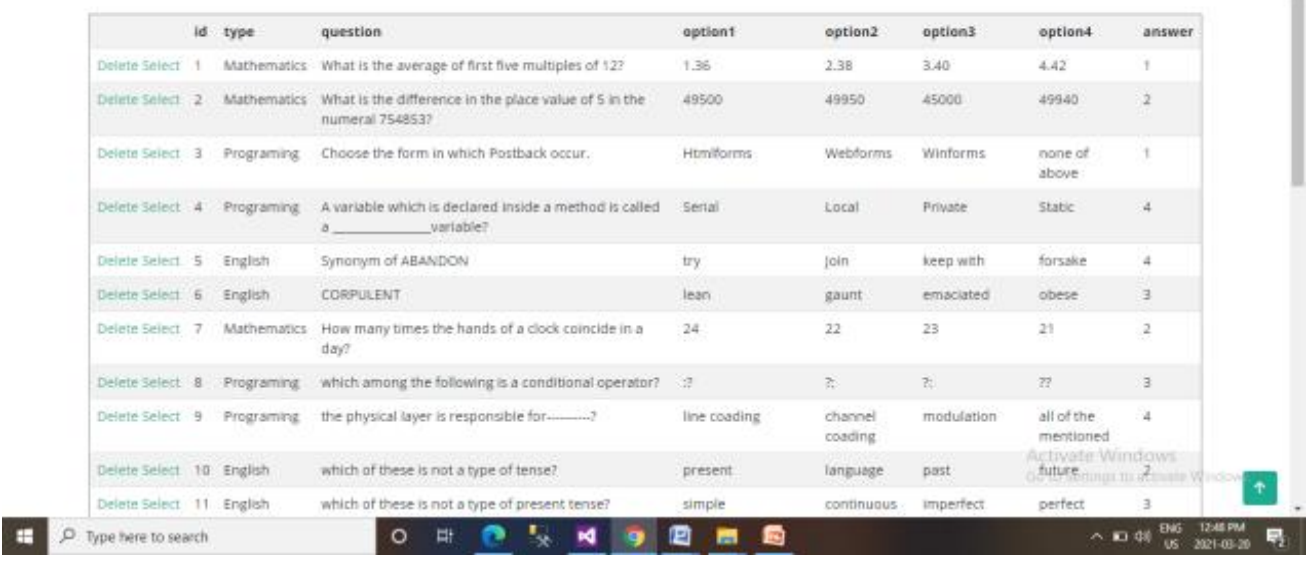

Figure: 1.8 - View Add A Question Page

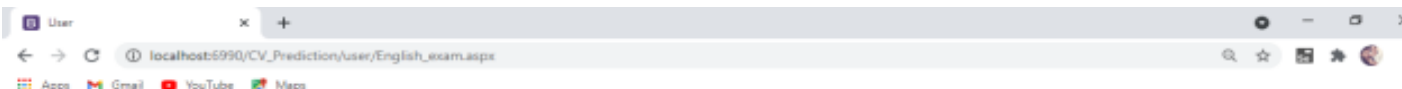

English Question

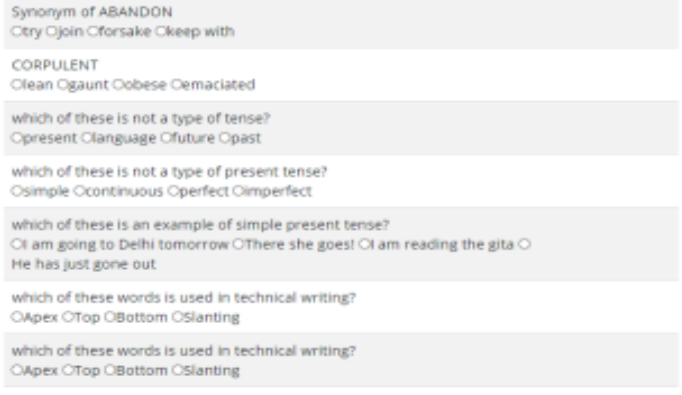

So type here to seach

Figure: 1.9 - Take A Test Page

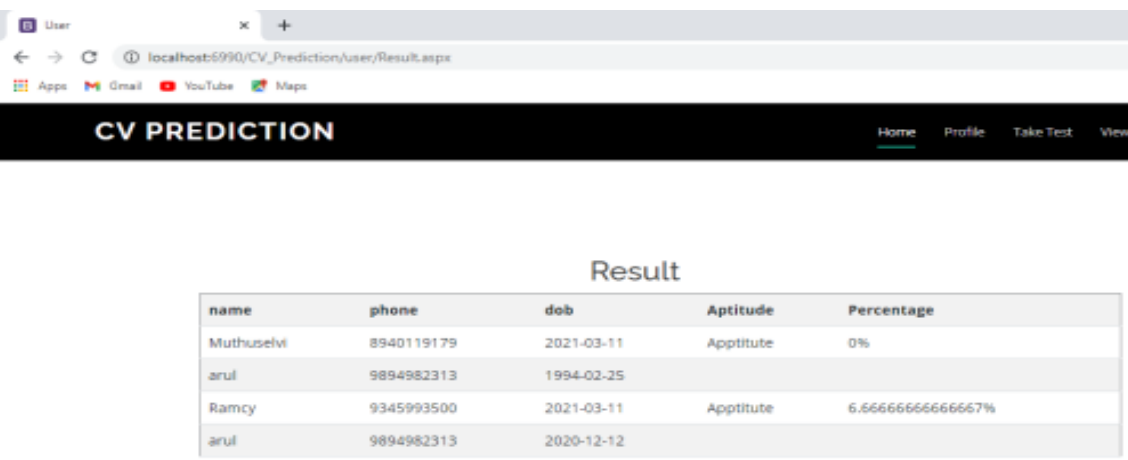

Figure: 1.10 View Result Page 


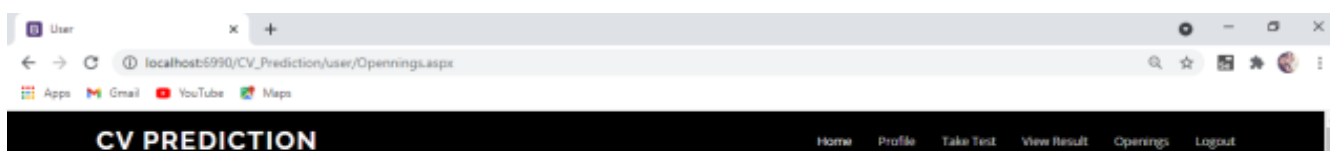

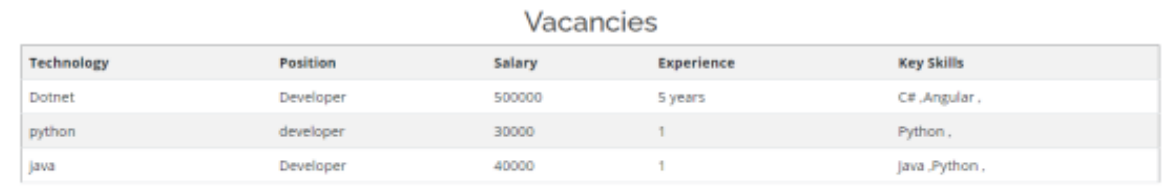

Figure: 1.11 - Opening Page
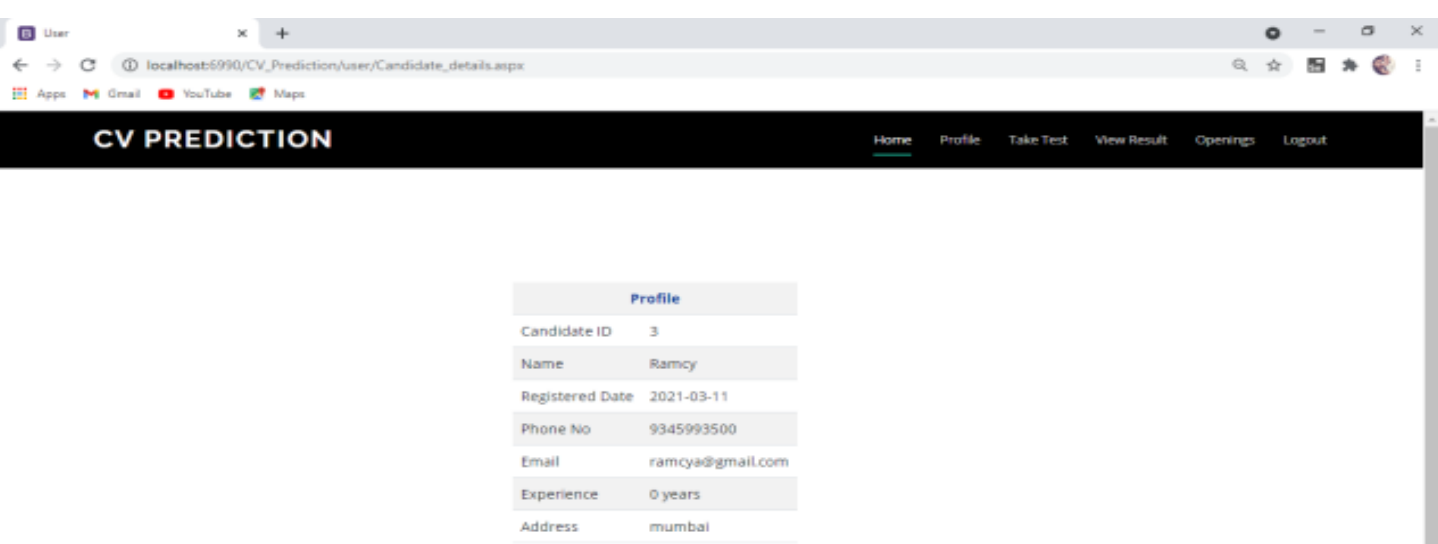

Figure: 1.12 - User Profile Page

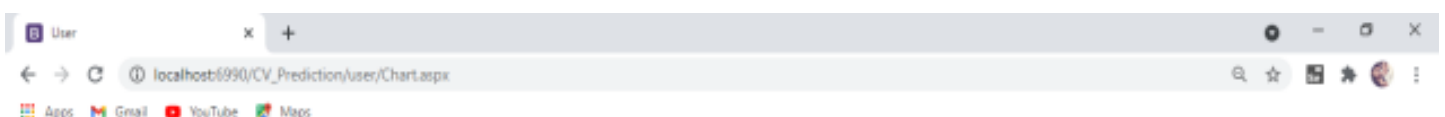

CV PREDICTION

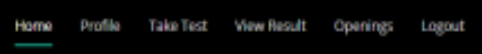


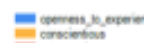
를

ocopylett Maxim. Al Rlate Reered Despres by opdicion

Figure: 1.13 - Chart Page 


\section{CONCLuSion}

This system will help the human resources department to select right candidate for particular job position, which in turn provide expert workforce for the organization. This system will help to get shortlisted CV's according to their ranking. Ranking is based on their test result and experience, qualification etc. This system will reduce work of the human resource department.

\section{FutUre ENHANCEMENT}

Further, we can alter the current framework to perform a conclusion examination of web-based life information.Some more arrangement calculations of AI can be incorporated to give much better functionalities.Further, the effectiveness and execution of the application can be tried and broke down.

\section{REFERENCES}

[1] F. Safia, N Ashaamitha Mathew.(2014) “”, International Journal of An integrated e-recruitment system for automated personality mining and applicant ranking.

[2]. S.dilini , P kusai(2017) "Personality based E- recruitment system , 29th Annual International Conference of the IEEE.

[3]. Allan Robey1, Kaushik Shukla2, KashishAgarwal(2018)SystemPersonality Prediction System through CV Analysis(2018). D Tantam, The machine as psychotherapist: impersonal communication with a machine. BJP synch Advances2017.

[4]. Barrick M. R. Mount. M. K proposed big five personality dimensions for any person in 1991 to determine the personality of any reason.

[5] Shruti Maheshwari, G. K. (2019). Personality Prediction through Curriculum Vitae Analysis Involving Password Encryption and Prediction Analysis. International Journal of Advanced Science and Technology, 28(16), $01-10$.

[6]. FoDRA - Nikolaos D. Almalis George A. Tsihrintzis, Aggeliki D. Strati , "A New Content-Based JobRecommendation Algorithm for Job Seeking andRecruiting", 2016.

[7] Data,Vishnu M Menon Computer Rahul Nath H A, "ANovel Approach to Evaluate and Rank Candidates in ARecruitment Process by Estimating EmotionalIntelligence through Social Media",May 2016.

[8] Manasi Ombhase, Prajakta Gogate, Tejas Patil "Automated Personality Classification Using DataMining Techniques" 10.13140/RG.2.2.35949.59363, 2017.

[9]. Amit Palve, Rohini D.Sonawane, Amol D. Potgantwar,"Sentiment Analysis of Twitter Streaming Data forRecommendation using, Apache Spark", InternationalJournal of Scientific Research in Network Security andCommunication, Vol.5, Issue.3, pp.99-103, 2017.

[10]. Huan Liu, Lei Yu, "Towards integrating feature selectionalgorithms for classification and clustering", IEEETransaction on Knowledge and Data Engineering,. 


\section{AUTHORS}

Ms.M.Muthuselvi doing final year MCA in Francis Xavier Engineering College

Mrs.D.Angeline Rangithamani is working as Assistant Professor and HOD in the department of Computer Applications, Francis Xavier Engineering College. She is having teaching experience of $12+$ years. Her area of intrest is Wireless Sensor Networks.

Ms.V.Abinaya doing first year MCA in Francis Xavier Engineering College.

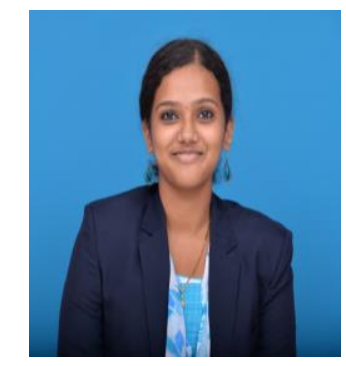

